\section{Application of enhanced thermal ratings to primary substation transformers}

\author{
Ian Elders ${ }^{1}{ }^{凶}$, Keith Bell ${ }^{1}$, Kevin Smith ${ }^{2}$, Alan Collinson ${ }^{2}$ \\ ${ }^{1}$ University of Strathclyde, Glasgow, UK \\ ${ }^{2}$ SP Energy Networks, Prenton, UK \\ $\bowtie$ E-mail: i.elders@strath.ac.uk
}

\begin{abstract}
The use of thermal modelling to increase the permissible load-carrying capability of distribution system transformers is attracting increasing interest. Many reported approaches calculate the rating of transformers in real time in response to system conditions. In this study, an experiment to validate and tune the parameters of such a thermal model is described, and explained how the results have been used to inform the inclusion of model-based seasonal 'enhanced' ratings in the network planning process.
\end{abstract}

\section{Introduction}

Expected changes in the nature of demand and generation connected to electricity distribution networks, notably the increased take-up of low-carbon technologies, such as electric vehicles, heat pumps and small-scale generation, present new challenges in the planning and operation of these networks. Traditional reinforcement methods tend to be expensive and time consuming to deploy, and risk either leaving stranded assets if reinforcement is undertaken ahead of expected demand, or network constraints if it is delayed. These are particular concerns, given that these low-carbon technologies can often be deployed in much shorter timescales than traditional network reinforcement but remain the subject of considerable uncertainty as to the extent and timescale of their deployment in any particular network.

Improvement of primary substation $(33 / 11 \mathrm{kV})$ transformer capacity is a particular issue in terms of cost, time to deploy and the increment in capacity delivered - an increase of capacity of 33-50\% (depending on the number of existing transformers at the constrained location) may be the only possible response to a relatively small capacity shortfall. However, many transformers supply load which varies cyclically, with a low overnight load, a daytime plateau and a short evening peak at which the capacity constraint is felt. A transformer whose peak load is close to its 'nameplate' rating is likely to be relatively lightly stressed most of the time, and therefore able to tolerate a mildly increased short-duration peak load. In addition, in the UK, most load peaks occur in winter, when ambient temperatures are low. Modelling the thermal behaviour of the transformer in response to actual load and weather patterns may allow the calculation of an increased peak capacity which can be supported without excessive insulation deterioration or risk of failure.

Use of dynamic or 'real-time' thermal rating of transformers has been reported by Unison Networks in New Zealand [1]. In this application, calculation of real-time ratings is closely integrated into the utility's information systems. In the UK, work towards dynamic rating of primary substation and distribution transformers has been reported as part of Western Power Distribution's FALCON project $[2,3]$. Both of these applications use relatively simple calculation models (IEC 60076-7 [4] and IEEE C57.91) to represent transformer behaviour.

The IEC 60076-7 model is adopted here as a relatively simple model requiring quite basic additional information beyond that currently used in the power system planning process. This is particularly important where the rating of mid-life transformers is to be evaluated since manufacturers' design and test records may be difficult or impossible to access. Furthermore, in contrast to other work considering real-time dynamic ratings, it was considered preferable to determine a small set of enhanced 'generally achievable' seasonal ratings which could be readily included in the existing planning process, rather than calculating dynamic ratings during transformer operation. This is discussed further later in the paper.

The 'Flexible Networks for a Low Carbon Future' innovation project led by SP Energy Networks [5] offered an opportunity to practically evaluate the use of transformer thermal models to deliver additional network capacity through enhanced thermal ratings, and to develop methods for their use in network planning as an alternative to traditional reinforcement. Experiments were undertaken on a selected primary substation transformer to evaluate its thermal behaviour under normal and increased loading conditions. The results were used to identify representative parameters for the IEC transformer thermal model, to determine suitable margins to allow for measurement and modelling error, and to assess the ability to support additional load beyond the nameplate rating.

In the following sections, we describe the experimental method used, including minimising the risk associated with deliberately increasing the load on the transformer beyond its 'nameplate' rating, and show and analyse the experimental results. Finally, we describe the resulting approach to use enhanced thermal ratings for transformers in the distribution network planning process.

\section{Experimental approach}

The transformer selected for the experiment, shown in Fig. 1, is located at Liverpool Road primary substation, and is rated at 7.5 MVA; no forced cooling is present. This substation has a single $33 / 11 \mathrm{kV}$ transformer, and is connected to two adjacent single-transformer substations via five $11 \mathrm{kV}$ cable circuits, which supply to a number of $11 / 0.4 \mathrm{kV}$ secondary substations. Under normal conditions, normally open switches part way along these cables divide the circuit load between Liverpool Road and the adjacent primary substations, as shown in Fig. 2.

The load on the Liverpool Road transformer was increased by closing each of the mid-feeder normally open points and opening the corresponding circuit breakers at the remote substations, as shown by the dashed arrows in Fig. 2 .

\subsection{Transformer and network monitoring}

Since the experiment was to be carried out on an in-service transformer, it was essential that the behaviour of the transformer 


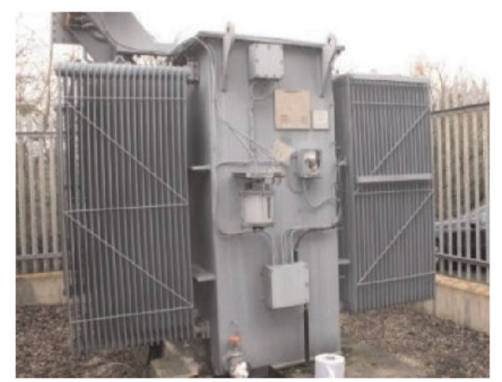

Fig. 1 Liverpool Road primary transformer

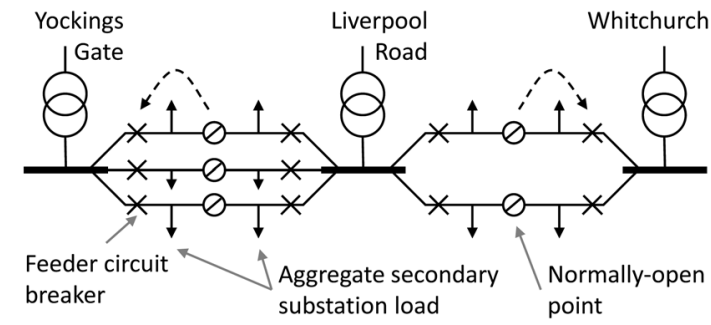

Fig. 2 Distribution network used in experiment

was closely monitored, so that any sign of distress could be detected and the experiment terminated without risk of interruption of supply to customers. Fig. 2 shows that the number of customers at risk to loss of the transformer would be larger than usual during the experiment. Furthermore, recording of the load and thermal behaviour of the transformer was required for subsequent analysis.

In Fig. 2, current at each primary substation transformer and each outgoing circuit breaker were reported in real time and recorded at 30 min intervals by the distribution management system (DMS). In addition, each transformer was equipped with a mechanical temperature indicator, whose contacts generated alarm and trip signals reported by the DMS at set temperatures. As part of the 'Flexible Networks' project, a supplementary monitoring device was installed at each substation recording current, active power and reactive power (among other values) on each circuit at $10 \mathrm{~min}$ intervals. The temperature monitor at Liverpool Road was replaced by an electronic indicator reporting temperature via the DMS, whose output was also recorded at $10 \mathrm{~min}$ intervals by the substation monitor. The network control engineer was thus able to observe the temperature of the transformer in real time, and able to reduce load if required to avoid over-temperature alarms or trips.

The electronic temperature monitor installed measures the oil temperature at the top of the transformer, and then applies a linear factor based on the measured C-phase current to estimate the winding temperature; the winding temperature only is reported via the DMS and monitoring systems to a precision of $1^{\circ} \mathrm{C}$. In the analysis described here, this calculation was reversed to recover the measured oil temperature.

In addition, weather stations were installed at Liverpool Road and Whitchurch primary substations; ambient temperature data from these locations was used as part of the transformer thermal modelling process.

\subsection{Preliminary analysis}

Prior to the experiment, and before installation of the additional monitoring devices described above, the transformer and associated plant (including bushings and cables) were physically examined. The associated plant was considered to be capable of supporting any reasonably achievable increased transformer loading. The expected thermal performance was calculated using a version of the IEC thermal model using general thermal parameters, as described in [6]. This calculation gave an expected rating for the measured load shape of 7.62 MVA.

Following installation of the temperature and load monitoring equipment, the thermal behaviour of the transformer was recorded under normal late-autumn loading conditions and compared with that of model when parameterised using 'specimen' values. As a result, the model parameter representing the oil temperature rise at rated losses was reduced from an initial value of 52 to $43^{\circ} \mathrm{C}$ to improve the correspondence between modelled and measured temperature. This allowed forecasting of the transformer's behaviour under increased load and to determine whether its temperature might reach an unacceptable level. An expected rating of 8.32 MVA was calculated at rated insulation temperature.

\subsection{Experimental and analytical procedure}

The experiment took place over an $\sim 84 \mathrm{~h}$ period, beginning on a Monday morning in late January. At the beginning of the experiment, the load on the transformer was rapidly increased over the course of a few minutes by sequentially transferring feeder load from adjacent substations, as described above. During this process, the transformer was closely monitored by control room staff using the additional monitoring data available through the DMS. This monitoring continued throughout the experiment, during which no signs of transformer distress were observed.

The network remained in this new configuration for the entire period of the experiment, and the load on the transformer was allowed to vary according to the usual pattern in this section of the network. At the end of the experiment, the network was returned to the 'usual' configuration, with the exception that, for operational reasons, the normally open point in one circuit was returned to a different point on the feeder from that at the start of the experiment. As before, this switching was undertaken in sequence over the course of a few minutes.

Following the conclusion of the experiment, transformer load and temperature data, and weather data were recovered from the substation monitoring systems. At that point, it was discovered that, owing to a failure of the monitoring systems, no load or transformer temperature data had been recorded from Liverpool Road substation for a $23 \mathrm{~h}$ period beginning $\sim 30 \mathrm{~min}$ prior to the start of the experiment. Two samples were also missing on the last afternoon of the experiment. It was decided to replace the missing load data with synthesised data based on preceding and subsequent weeks for the purposes of model initialisation (as discussed below), but to exclude these periods from the analysis of the results. The resulting time series of load for the period during and surrounding the experiment is shown in Fig. 3.

The peak load observed on the transformer during the experiment was $9.11 \mathrm{MVA}$, or $121 \%$ of nameplate rating, while the minimum load was 5.06 MVA. It can be seen from Fig. 3 that the minimum load is comparable to the peak load experienced under 'normal' conditions. The ambient temperature during the experiment varied between 0 and $10^{\circ} \mathrm{C}$, with the last $24 \mathrm{~h}$ of the period being noticeably colder than the first three days.

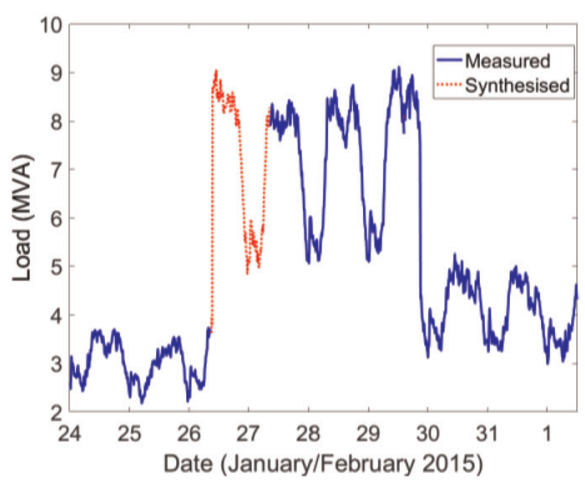

Fig. 3 Modelled load on Liverpool Road primary substation 
Table 1 Parameters of interest

\begin{tabular}{lc}
\hline Symbol & Parameter \\
\hline$\Delta \theta_{\text {or }}$ & oil temperature rise over ambient at rated losses \\
$\tau_{\mathrm{o}}$ & oil temperature time constant \\
\hline
\end{tabular}

An implementation of the IEC60076-7 thermal model was constructed using Matlab Simulink, such that time series of ambient temperature and load measurements could be used to produce corresponding series of modelled oil and hotspot temperature values - this will be referred to as a 'model run'. The model was initially parameterised using values from the preliminary analysis and winding temperature parameters adjusted to correspond to the calculation method used by the winding temperature monitor; some of these parameters were further refined in the light of actual measurements, as discussed below.

For each model run, the model was initialised using measured and synthesised load data from the beginning of the period shown in Fig. 3 until a point $8 \mathrm{~h}$ after the end of the synthesised data and discarding the results - a total of $94 \mathrm{~h}$ of data, significantly in excess of the expected time constants of the model. It was thus expected that effects of initialisation and inaccuracy in the synthesised data would be eliminated.

The measured and modelled temperature data for the period from the end of initialisation until the end of the experiment were then compared, and certain parameters of the model were adjusted in sequence to improve the correspondence between measured and modelled behaviour. The parameters of interest are listed in Table 1.

Given that the winding temperature is calculated from the oil temperature and load rather than being measured, corresponding winding and hotspot parameters were not considered susceptible to calculation; hence, the hotspot temperature rise parameter $\Delta \theta_{\mathrm{hr}}$ was set to the correction factor used by the temperature monitor, further corrected from a winding to hotspot value as set out in IEC 60076-7 (for a final value of $19.5^{\circ} \mathrm{C}$ ). The winding time constant remained at the value suggested in the IEC standard.

Given the relatively small amount of data available, and the limited dynamic behaviour observability resulting from the data loss, no other parameters were optimised.

\section{Results and analysis}

\subsection{Temperature measurements}

The oil and winding temperatures recorded from the transformer are shown in Fig. 4.

The zero values corresponding to the two periods of missing data can be seen starting on 26 January and, briefly, on 29 January. A number of features are circled in which the transformer's temperature appears to change suddenly. As discussed further

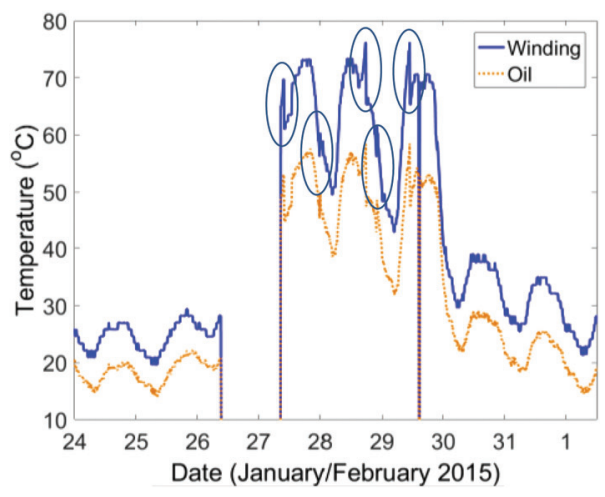

Fig. 4 Recorded transformer temperatures
Table 2 Final values of parameters

\begin{tabular}{lcc}
\hline Parameter & IEC value & Fitted value \\
\hline$\Delta \theta_{\mathrm{or},}{ }^{\circ} \mathrm{C}$ & 52 & 45.3 \\
$\tau_{\mathrm{o}, \min }$ & 210 & 388 \\
\hline
\end{tabular}

below, these are not considered to represent actual changes in the transformer's general thermal state, and they and the periods around them were excluded from the analysis.

\subsection{Fitting of model parameters}

Following each run of the model, one of the two parameters of interest was adjusted so as to reduce the sum of squares of the differences between measured and modelled oil temperature. Once a minimum had been found for one parameter, the process was repeated for the other, until no improvement could be found by adjustment of $0.1^{\circ} \mathrm{C}$ and $1 \mathrm{~min}$ in the parameters. Final values of these parameters are given in Table 2 .

It is noted that $\Delta \theta_{\text {or }}$ is somewhat less than that suggested by IEC60076-7, while $\tau_{\mathrm{o}}$ is significantly longer. This suggests that this transformer is rather less responsive to heavy loading and sudden changes in load (such as might be experienced in response to the trip of a parallel transformer) than would be suggested by use of the suggested parameters. However, $\Delta \theta_{\text {or }}$ is much closer to the value calculated from the preliminary analysis.

\subsection{Observations on transformer behaviour}

Observed transformer oil temperature is compared with that calculated using the fitted model in Fig. 5.

It appears that there is a reasonable correspondence between the measured and modelled temperature, although with some behavioural differences which we consider may be related to limitations of the model. The maximum recorded and modelled oil temperatures were 58.5 and $55.2^{\circ} \mathrm{C}$, respectively; corresponding hotspot temperatures were 78.7 and $79.8^{\circ} \mathrm{C}$. The largest underestimated daily peak hotspot temperature was $0.2^{\circ} \mathrm{C}$.

3.3.1 Model limitations: Some clear divergences between modelled and measured behaviour appear in Fig. 5. The first is the sequence of sudden changes of measured transformer temperature circled in Fig. 4. It appears that the measured oil temperature suddenly falls each day while the temperature is rising towards or is close to its daily maximum and suddenly rises during the cooling period after the daily peak. The measurements can be divided into 'low temperature' and 'high temperature' operating regimes. These steps (of up to $11^{\circ} \mathrm{C}$ in a 10 min measurement interval) are significantly larger than that can be explained by unobserved changes in load and ambient temperature. They were only observed during the experiment, and do not appear in measurements taken under normal operating conditions. We suggest that they may reflect changes in the oil-flow pattern within the transformer at high temperature and load, such that the

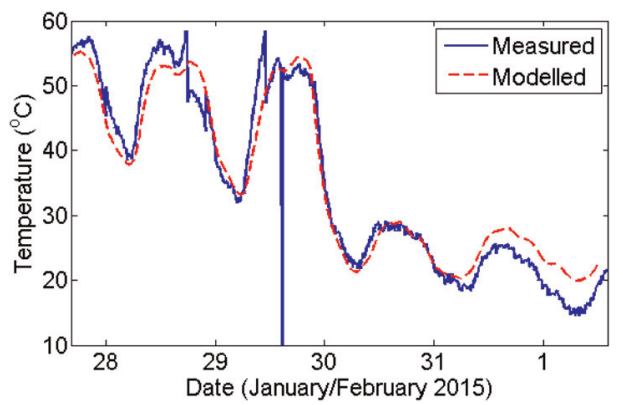

Fig. 5 Measured and modelled oil temperature 
temperature sensor is located either in a stream of heated oil emanating from the windings, or in cooler oil more representative of conditions at the top of the transformer. Although the transformer tested is naturally cooled, we note that rapid changes in oil flow might also be expected from operation of temperature-controlled force-cooling equipment.

For the intended application of enhanced thermal ratings, we believe that it is not necessary to model this behaviour in detail, and that conservative selection of model parameters will account for any risk of unexpectedly high transformer temperature. However, further investigation may be worthwhile to optimise the location of the temperature probe to achieve consistent measurements in line with the intent of the thermal model.

The second difference in behaviour relates to the period following the end of the experiment. The modelled temperature behaviour settles quickly to a cyclic pattern about a reasonably constant mean value. The measured temperature shows a clear but gradual decline over the days following the experiment: limitations on the availability of data prevented a full characterisation of the duration of this decline. It is suggested that this behaviour relates to a slow release of stored thermal energy, perhaps from the transformer core, which had accumulated during the period of high loading. The failure to record the early part of the experiment prevents investigation of any corresponding behaviour at the onset of high loading as energy accumulated in the transformer. It is, however, likely that the overall effect would be that of a further, unmodelled time constant to the transformer behaviour that would slow its reaction to sudden load changes.

It is perhaps unsurprising that the relatively simple model specified by IEC 60076-7 does not represent detailed transformer behaviour with complete fidelity. It also appears that measurement arrangements which are satisfactory under normal conditions may not be entirely suitable under extreme loading. However, we consider that, given that the objective of the work is not to press the transformer to its ultimate capability, these limitations can be accommodated by allowing suitable margins in the model parameters and permissible hotspot temperature. Considering sources of experimental error in our results as well, we suggest a margin of $3^{\circ} \mathrm{C}$ in $\Delta \theta_{\text {or }}$ and $8.5^{\circ} \mathrm{C}$ in maximum temperature (mainly associated with the sudden temperature changes). Use of more conservative thermal parameters may permit a lower temperature margin.

\section{Network planning application}

The results of the experiment and analysis described in this paper demonstrate that consideration of the thermal behaviour of transformers in relation to actual patterns of load and ambient temperature can release useful additional load-carrying capability. Investment in network reinforcement in response to increasing load might therefore be deferred or avoided.

SP Energy Networks is incorporating thermal modelling of transformers into its network planning processes, invoked when a deficiency in transformer capacity is observed as part of the regular process of reviewing network capacity in comparison to existing and forecast load. A spreadsheet implementation of the IEC thermal model is then used to calculate a set of seasonal 'enhanced ratings', based on scaled historical load patterns and assumed seasonal ambient temperatures, which can be achieved without exceeding the rated insulation temperature of the transformer. This enhanced rating approach has a number of practical advantages over dynamic ratings calculated in real time during operation:

- The network planning process is already adapted to the use of seasonal overhead line ratings.

- Modelling of thermal performance is carried out offline. Upgrading of online operational systems to calculate ratings in real time is avoided, enabling faster realisation of increased capacity.
- The need to forecast, within the long-term planning task, real-time ratings achievable in the future is also avoided.

Where the enhanced ratings approach shows that a benefit can be achieved, deferral of investment can be achieved at relatively low cost and without the necessity for a prior programme of load tests in each case. Further, as discussed below, more capacity may be released later through improved monitoring and modelling of transformer behaviour. It is of course possible that the initial modelling will reveal that the transformer loading pattern does not permit rating enhancement (such as for example a transformer with a largely constant load). In such cases, planning of traditional reinforcements is undertaken.

The traditional approach to the management of distribution transformer condition and the risk of unexpected failure is based on periodic inspection and conservative rating assumptions. The use of transformer thermal modelling to enhance a transformer's rating replaces some of these traditional assumptions with more accurate information. Some uncertainty remains in respect of measurement accuracy, and patterns of future load and weather. In selecting a seasonal enhanced rating, some uncertainty margin should thus be included at the modelling stage e.g. the use of conservative assumptions about ambient temperature, or use of the suggested model parameters from the IEC standard, which, for the transformer tested, appear conservative.

More assurance of the suitability of the calculated ratings is provided by improved knowledge and monitoring of transformer condition and behaviour once the potential for enhanced thermal rating has been identified. Recommendations at this stage include the following:

- Physical inspection of the transformer (as described in [6]) to ensure that its condition and remaining life are suitable for enhanced rating.

- Where economically viable, the condition of the transformer may be improved by refurbishment.

- Installation of remote temperature monitoring and recording capability for the transformer.

- Regular review and reanalysis of the thermal behaviour of the transformer, and comparison with recorded temperature data.

It is noted that this latter activity allows for an analysis process similar to that described in this paper, which would yield better knowledge of actual transformer thermal parameters. We note that an acceptable estimate of thermal parameters at high load can be obtained under normal winter loading. These parameters could be recorded and introduced into the capacity calculation, potentially revealing further available capacity headroom which could be used to accommodate future load growth.

There are a number of locations in SP Energy Networks' distribution system at which enhanced transformer thermal ratings are being pursued as an alternative to traditional reinforcement.

\section{Conclusions}

Modelling of the thermal behaviour of transformers offers opportunities to defer or avoid costly distribution system reinforcement. The experiment and analysis undertaken at Liverpool Road shows that this capability exists in practice, and can be exploited on the basis of conservative assumptions about the behaviour of the transformer. Furthermore, once an enhanced rating regime has been established for a transformer, measurement and modelling of the actual thermal behaviour can allow assumptions in the thermal model to be refined, releasing more capacity. A good estimate of the transformer's thermal behaviour can be obtained under normal peak load conditions without the need for testing under extreme load.

Use of seasonal 'enhanced' thermal ratings allows this additional capacity to be more easily incorporated into existing business processes than 'real-time' thermal ratings. Offline modelling of the 
thermal behaviour of the transformer in this approach is integrated into existing network capacity review tasks using a spreadsheet-based tool. Modification of operational systems can be restricted to use of pre-calculated seasonal ratings for relevant transformers (as is already done for overhead lines) and simple remote monitoring of those transformers. The approach can therefore be quickly deployed to defer reinforcement to relieve anticipated overloads, thereby yielding cost savings for DNOs and customers.

\section{Experimental data}

Measurement and associated data used in the work described in this paper is available [7]

\section{Acknowledgments}

The 'Flexible Networks for a Low Carbon Future' project was funded through the Low Carbon Networks Fund. The authors acknowledge the collaboration of colleagues at SP Energy
Networks in the experiments described in this paper. The spreadsheet implementation of the IEC model was developed in collaboration with TNEI Services Ltd.

\section{References}

1 Jalal, T.S.: "Case study: implementation of dynamic rating for transformers in a distribution network'. Innovative Smart Grid Technologies Conf., Washington DC, February 2014

2 Yang, J., Strickland, D.: 'Thermal modelling for dynamic transformer rating in low carbon distribution network operation'. 7th IET Int. Conf. Power Electronics, Machines Drives, Manchester, April 2014

3 Chittock, L., Yang, J., Strickland, D., et al.: 'Distribution network transformer thermal modelling parameter determination for dynamic rating applications'. 8th IET Int. Conf. Power Electronics, Machines Drives, Glasgow, 2016

4 International Electrotechnical Commission: 'Loading guide for oil immersed power transformers', IEC Standard 60076-7, 2005

5 'Flexible networks for a low carbon future'. Available at http://www. spenergynetworks.co.uk/pages/flexible_networks_for_a_low_carbon_future.asp

6 Meijer, S., de Wild, F., Boone, W., et al.: 'Dynamic rating to support safe loading of distribution transformers'. Proc. CIRED Conf., Lyon, June 2015

7 'SP energy networks 'flexible networks' electricity distribution system measurements'. Available at http://pure.strath.ac.uk/portal 